\title{
The Discrete Rational Cubic Spline Interpolator
}

\section{Y.P. Dubey, Jyoti Tiwari}

\begin{abstract}
The problem of Discrete $C^{2}$ Rational Cubic Spline has been proposed and Error bound obtained. The Discrete Rational Method have unique representation.
\end{abstract}

Keywords: Rational, Discrete, Cubic, Shape Preserving, Error bounds, Interpolation Parameter.

\section{INTRODUCTION}

$\mathrm{R}$ ational Cubic spline with one free parameter useful to obtained positive and convex curve from positive and convex data respectfully. discrete spline obtained by using differences in place of derivative . Rana and Dubey [3] constructed discrete cubic spline. Duen [4] study of rational interpolation with functional values .To find design and shape of curve rational and discrete rational cubic spline are applicable So many author studies for shape preservation of curve ( see [6], [7], [8] ,[9] [10],). Rational discrete cubic spline preserve convexity and positivity but simple spline not preserve both. Duan et.al. [4]have found shape of curves by using rational spline and obtained condition that curves lie to above, blow or between the straight lines . In this paper authors assumed suitable values of parameters to obtain $\mathrm{C}^{2}$ Conversion Curve and the scheme work for uniform mesh. Hussain et.al. [5] investigate a rational cubic function which was used to achieve designs for shape data. They found relation on free parameters in the description of $\mathrm{C}^{1}$ rational cubic function to obtain desire shapes of the data. In this paper we have using different values of free parameter to obtained curves for uniform and non uniform case. We have developed discrete rational cubic spline with one free parameter

\section{Remark 1.1}

When $h_{i} \rightarrow 0$, we may obtained $\mathrm{C}^{2}$ rational cubic spline with two parameters this gives particular case. M.Z. Hussain et.al. [5].

Sarfaraz [6], Abbas [7], Duan [4] and Bao [9] error bound obtained by sub interval in our paper error bounds obtain one time full interval $[0,1]$.

Error bound obtained in our paper is very accurate and minimum but other paper error bound get maximum inaccurate.

Manuscript received on August 31, 2021.

Revised Manuscript received on September 23, 2021.

Manuscript published on September 30, 2021.

* Correspondence Author

Dr. Yadvendra Prasad Dubey*, HOD and Professor, Govt Kundam College Jabalpur (M.P) India

Smt Jyoti Tiwari, Assistant Professor, Department of Mathematics, Shri Ram Institute of Science and Technology, Jabalpur (M.P) India.

(C) The Authors. Published by Blue Eyes Intelligence Engineering and Sciences Publication (BEIESP). This is an open access article under the CC BY-NC-ND license (http://creativecommons.org/licenses/by-nc-nd/4.0/)

(iv) When differences is given then our scheme is beneficial but Broadlieand Butt[11] Abbas et.al [7].Sarfaraze et.al [6].and so many paper methods are not useful.

\section{C ${ }^{2}$ DISCRETE RATIONAL CUBIC FUNCTION}

Now $\mathrm{s}(x)$ is defined as

$$
s(x)=s\left(x_{i}\right)=\frac{p_{i}(\theta)}{q_{i}(\theta)}=\frac{\sum_{i=0}^{g} w_{i}(1-\theta)^{s-i} \theta^{i}}{q(\theta)}
$$

with..(2). $x_{i+1}-x_{i}$

$\theta=\frac{\left(x-x_{i}\right)}{h_{i}}, i=1,2, \ldots . . n-1$

and $w_{0}=f_{i}$

$w_{1}=\left[h_{i} d_{i}\left(h_{i}^{2}-\left(v_{i}-1\right)\right)^{2} h_{i}^{2}-f_{i}\left\{2\left(h^{2}-h_{i}^{2}\right)-\left(h_{i}^{2}+3 h^{2}\right) v_{i}\right\}\right.$

$+\frac{\left.w_{2} h^{2} h_{\mathrm{i}} v_{\mathrm{i}}-v_{\mathrm{i}} h_{\mathrm{i}+1} h^{2}\right]}{\left(h^{2}+h^{2}\right)+2 h^{2} v_{\mathrm{i}}}$

$w_{2}=\left[-d_{i+1} h_{i}\left\{\left(h_{i}+v_{i} h_{i}\right)^{2}-v_{i}^{2} h^{2}\right\}-h_{i} h^{2} v_{i} h_{i}\right.$

$+v_{\mathrm{i}} h_{\mathrm{i}+1}\left\{h_{\mathrm{i}}^{2}+3 h_{\mathrm{i}}^{2} h\right\}$

$\left.\left.2 h^{2} v_{i}+3 h_{\mathrm{i}}^{2} h+2 v_{\mathrm{i}} h_{\mathrm{i}}^{2}\right\}\right]$

$\left[2 h^{2} h_{\mathrm{i}}-h^{2} h_{\mathrm{i}} v_{\mathrm{i}}-v_{\mathrm{i}} h_{\mathrm{i}}^{\mathrm{a}}\right]$

$w_{3}=v_{i} f_{i+1}$

$q_{i}(\theta)=(1-\theta)^{2}+\left(1+v_{i}\right) \theta(1-\theta)+v_{i} \theta^{2}$

Where $v_{i}$ is the shape parameter used to control the shape of the interpolation and Let $D_{h}^{(2)} s(x)$ the second differences with respect to $x$ and $d_{i}=D_{h}{ }^{(1)} s\left(x_{i}\right)$ denote first differences value at knots $x$, then $\mathrm{C}^{2}$ splining constrains :

$$
\begin{aligned}
& s\left(x_{i}\right)=f_{i} ; s\left(x_{i+1}\right)=f_{i+1} \\
& D_{h}^{(1)} f\left(x_{i}\right)=d_{i}, D_{h}^{(1)} f\left(x_{i+1}\right)=d_{i+1} \\
& D_{h}^{(2)}\left(x_{i}+\right)=D_{n}^{(2)}\left(x_{i}-\right), i=1,2, \ldots . . n-1
\end{aligned}
$$

Where $D_{h}{ }^{(1)}(x)=\{f(x+h)-f(x-h)\} / 2 h, \quad D_{h}^{(2)} f(x)=[f(x+h)$ $-2 f(x)+f(x-h)] / h^{2}$.

Using continuity of second derivative we get

$$
\begin{gathered}
\frac{h_{i}, d_{i-1} v_{i-1}\left[h^{2}\left(1-v_{i-1}\right)+h_{i-1}^{2} v_{i-1}^{2}\right]}{\left\{-\left(1-v_{i-i}\right)^{2} h^{2}+v_{i-1}^{2} h_{i-1}^{2}\right\}} \\
+\frac{d_{i}\left[\left\{h_{i} v_{i-1}\left\{h^{2}\left(1-v_{i-1}\right) v_{i-1}+h_{i-1}^{2} v_{i-1}+h_{i-1}^{2} v_{i-1}^{2}\right\}\right.\right.}{\left[-\left(1-v_{i-1}\right)^{2} h^{2}+v_{i-1}^{1} h_{i-1}^{2}\right]}
\end{gathered}
$$




$$
\begin{aligned}
& \left.+h_{i-1} d_{i}\left\{-2\left(1-v_{i}\right)^{2} h^{2}+\left(h_{i}^{2}+h^{2}\right)\right\}\right] \\
& +\frac{+v_{i} h_{i-1} d_{i+1}\left[h^{2}\left(1-v_{i}\right)+h_{i}^{2}\right]}{\left[-\left(1-v_{i}\right)^{2} h^{2}+h^{2}\right]} \\
& \quad=F_{i} * i=1,2, \ldots \ldots . . n-1 .
\end{aligned}
$$

Where

$$
\begin{aligned}
& \quad F_{i}^{*} \\
& =h_{i-1}\left(1+2 v_{i}\right) \\
& {\left[\left(1-v_{i}\right)\left(h^{2}+h_{i}^{2}\right)+2 h_{i}^{2}+2 h_{i}^{2}\right] \Delta_{i}} \\
& +h_{i} v_{i-1}\left(v_{i-1}+2\right) \\
& {\left[\left(h_{i-1}^{2}+h^{2}\right)\left(1-v_{i-1}\right) v_{i-1}+2 h_{i-1}^{2} v_{i-1}^{2}\right] \Delta_{i-1}} \\
& {\left[\left(1-v_{i-1}\right)^{2} h^{2}+v_{i-1}^{2} h_{i-1}^{2}\right]}
\end{aligned}
$$

Where $\Delta_{i}=\left(f_{i+1-} f_{i}\right) / h_{i}$. Different parameter $d_{i}$ and $d_{i-1}$ determinate by end conditions.

Since the system of linear equation is diagonally dominant for all $v_{i}>0$, it has a unique solution for the difference parameters $d_{i}$ s. We can find solution of above equation.

Remark 2.1: When $h \rightarrow 0$, the Rational Discrete cubic spline reduce to rational cubic spline.

Remark 2.2: Without shape parameter when $v_{i}=1$ so the rational discrete cubic spline reduce to discrete cubic spline.

\section{POSITIVITY OF RATIONAL DISCRETE CUBIC SPLINE FUNCTION}

Let

$\left\{x_{i}, f_{i}\right\}, i=1,2, \ldots . n, x_{1}<x_{2}<\ldots \ldots<x_{n}$ and

$f_{1}>0, f_{2}>0 \ldots \ldots . f_{n}>0$ it is required to construct a positive interpolant $s(x)$. The rational discrete cubic function (1) is positive if $p_{i}(\theta)$ and $q_{i}(\theta)$ both positive, since $q_{i}(\theta)>0, \forall v_{i}>0$.

Now $p_{i}(\theta)$ can be rewritten as follows: $p_{i}(\theta)=a_{1} \theta^{3}+b_{i} \theta^{2}+c_{i} \theta+d_{i}$.

Where

$$
\begin{aligned}
& a_{i}=w_{1}-w_{0}-w_{2}+w_{3} \\
& b_{i}=3 w_{0}-2 w_{1} \\
& c_{i}=-3 w_{0}+w_{1}+w_{2} \\
& d_{i}=w_{0} . \\
& \text { So } a_{i}+b_{i}+c_{i}+d_{i} \\
& =w_{3}=v_{i} f_{i+1}>0 .
\end{aligned}
$$

\section{Theorem 3.1:}

The $C^{2}$ Rational Discrete Cubic function (1.1) is positive in each interval $\left[x_{i}, X_{i+1}\right]$ if the shape parameter $v_{i}$ satisfy following constraints

$v_{i}>0$

and $w_{3}=v_{i} f_{i+1}>0$.

\section{ERROR BOUNDS}

For a given $h>0$, we introduce the set $R_{h a}=\{a+j h: j$ is an integer $\}$ and define a discrete interval as follows: $[a, b]_{h}=[a, b] \cap R_{h a}$

For a function $f$ and three disjoint points $x_{1}, x_{2}, x_{3}$ in its domain. The first and second divided differences are defined by

$$
\begin{aligned}
& {\left[x_{1}, x_{2}\right]_{f}=\frac{\left\{f\left(x_{1}\right)-f\left(x_{2}\right)\right\}}{\left(x_{1}-x_{2}\right)}} \\
& \text { and }\left\{x_{1}, x_{2}, x_{3}\right\}_{f}=\frac{\left\{\left[x_{1}, x_{2}\right] f-\left[x_{2}, x_{3}\right] f\right\}}{\left(x_{1}-x_{3}\right)} \text { respectively. }
\end{aligned}
$$

Now we write $f^{(2)}$ for $D_{h}^{(2)} f$ and the modulus of continuity of $f$ is $w(f, p)$.

And $\quad\|f\|=\max _{x \in[a, b]_{h}}|f(x)|$ discrete norms of a function $f$ over the interval $[a, b]_{h}$

To obtained error bound we have to state following

\section{Theorem 4.1:}

Let $\mathrm{s}(\mathrm{x}, \mathrm{h})$ be the unique periodic discrete Rational cubic spline interpolant $f$ under the assumption of condition (2.3) . Then over the discrete interval $[a, b]_{h}$.

$$
\|e(x)\| \leq p k(h) w\left(D_{h}^{(1)} f, p\right)
$$

Where $\left.(k(h))^{4}\right)_{1}^{1)}$ some function of $h$ defined earlier and $w(f$, $p$ ) is the discrete modules of the continuity of $f$.

In order to show the convergence of the discrete rational spline. We shall need the following Lemma due to Lyche [12].

Lemma 4.1 : Let $\left\{a_{j}\right\}_{j=1}^{m}$ and $\left\{b_{j}\right\}_{j=1}^{n}$.be given sequence of non negative real number such $\sum_{j=1}^{m} a_{j}=\sum_{j=1}^{n} b_{j}$. Then for any real valued function $f$, defined on a discrete interval $[0,1]$ we have

$$
\begin{gathered}
\left|\sum_{j=1}^{m} a_{j}\left[x_{j}, x_{j, 1}-x_{j, k}\right]_{f}-\sum_{j=1}^{n} b_{j}\left[y_{j 0}, y_{j, 1} \ldots . . y_{j n}\right]_{f}\right| \\
\leq \mathrm{w}\left\{\mathrm{D}_{\mathrm{h}}{ }^{(\mathrm{k})} \mathrm{f},|1-\mathrm{kh}|\right\} \sum \mathrm{a}_{\mathrm{j}} / \mathrm{k} !
\end{gathered}
$$

Where $\mathrm{x}_{\mathrm{jk}}, y_{j k} \in[0,1]_{h}$ for rational values of $\mathrm{j}$, k. Replacing $m_{\mathrm{i}}$ by $D_{h}^{(1)} e\left(x_{i}\right)$ in Equation (2.4).

We have

$$
e(x)=v_{i}, h_{i-1} e_{i-1} \frac{\left[h_{i}^{2}\left(1-v_{i-1}\right)+h_{i-1}^{2} v_{i-1}^{2}\right]}{\left[-\left(1-v_{i-1}\right)^{2} h^{2}+h_{i-1}^{2} v_{i}^{2}\right]}
$$

$$
+\left\{e _ { i } \left[h_{i}\left\{h^{2}\left(1-v_{i-1}\right)+h_{i-1}^{2}\left(1+v_{i-1}\right) v_{i-1}\right\}\right.\right.
$$




$$
\begin{aligned}
& \left.\frac{+h_{i-1}\left\{-\left(1-v_{i}\right)\left(h^{2}+h^{2}\right)+2 h_{i}^{2}\right\}}{\left\{-\left(1-v_{i}\right)^{2} h^{2}+h_{i}^{2}\right\}}\right] \\
& \frac{+e_{i+1}\left[v_{i} h_{i-1}\left[\left(h^{2}\right)\left(1-v_{i}\right)+h_{i}^{2}\right]\right.}{\left[-\left(1-v_{i}\right)^{2} h^{2}+h_{i}^{2}\right]}
\end{aligned}
$$$$
\frac{h_{i-1}\left(1+2 v_{i}\right)\left[-\left(1-v_{i}\right)\left(h^{2}+h_{i}^{2}\right)+2 h_{i}^{2}\right]}{\left[-\left(1-v_{i}\right)^{2}+h_{i}^{2}\right]}
$$

$\frac{\Delta_{i}+h_{i} v_{i-1}\left(v_{i-1}+2\right)\left[\left(h_{i-1}^{2}+h^{2}\right)\left(1-v_{i-1}\right) v_{i-1}+2 h_{i-1}^{2} v_{i-1}^{2}\right]}{\left[-\left(1-v_{i-1}\right)^{2} h^{2}+v_{i-1}^{2} h_{i-1}^{2}\right]} \Delta_{i-1}$

$-v_{i-1} h_{i} f_{i-1}^{1}\left[h^{2}\left(1-v_{i-1}\right) v_{i-1}+h_{i-1}^{2} v_{i-1}^{2}\right]$

$$
\left[-\left(1-v_{i-1}\right)^{2} h^{2}+h_{i-1}^{2} v_{i-1}^{2}\right]
$$

$$
\begin{gathered}
f_{i}^{[1]}\left[h_{i}\left\{h^{2}\left(1-v_{i-1}\right)+h_{i-1}^{2}\left(1+v_{i-1}\right) v_{i-1\}}\right\}\right. \\
\frac{\left.+h_{i-1}\left\{-\left(1-v_{i}\right) h^{2}+2 h_{i}^{2}\right\}\right]}{\left[-\left(1-v_{i}\right)^{2} h^{2}+h_{i}^{2}\right]}
\end{gathered}
$$$$
\frac{v_{i} h_{i} f_{i+1}^{\{1\}}\left[-h^{2}\left(1-v_{i}\right)+2 h_{i}^{2}\right]}{\left\{-\left(1-v_{i}\right)^{2} h^{2}+h_{i}^{2}\right\}}
$$

After using from Lyche [12] formula we get,

$$
\frac{\sum a_{i}=h_{i-1}\left(1+2 v_{i}\right)\left[-\left(1-v_{i}\right)^{2}\left(h^{2}+h_{i}^{2}\right)+2 h_{i}^{2}\right]}{\left[-\left(1-v_{i}\right)^{2} h^{2}+h_{i}^{2}\right]}
$$$$
\frac{+h_{i} v_{i-1}\left(v_{i-1}+2\right)\left[\left(h_{i-1}^{2}+h^{2}\right)\left(1-v_{i-}\right) v_{i-1}+2 h_{i-1}^{2} v_{i-1}^{2}\right]}{\left[-\left(1-v_{i-1}\right)^{2} h^{2}+v_{i-1}^{2} h_{i-1}^{2}\right]}
$$$$
=\sum b_{j}
$$

Where $x_{10}=x_{i}+h$

$$
\begin{aligned}
& x_{20}=x_{i} \\
& x_{30}=x+h
\end{aligned}
$$

Where $x_{10}=y_{30}=x_{i+1}+h$

$$
\begin{aligned}
& x_{11}=y_{31}=x_{i}-h \\
& y_{20}=x_{20}=x_{i}+h \\
& y_{21}=x_{21}=x_{i}-h \\
& x_{30}=y_{1-0}=x_{i-1}+h \\
& x_{31}=y_{11}=x_{i-1} h
\end{aligned}
$$

and

$$
a_{1}=\frac{h_{i-1}\left(1+2 v_{i}\right)\left(-\left(1+v_{i}\left(h^{2}+h_{i}^{2}\right)+2 h_{i}^{2}\right)\right.}{\left[-\left(1-v_{i}\right)^{2}+1\right]}
$$

$a_{2}=\frac{h_{i} v_{i-1}\left(v_{i-1}+2\right)\left[\left(h_{i-1}^{2}+h^{2}\right)\left(1-v_{i-1}\right)+2 h_{i-1}^{2} v_{i-1}^{2}\right]}{\left[-\left(1-v_{i-1}\right)^{2}+h^{2}+v_{i-1}^{2} h_{i-1}^{2}\right]}$

$$
b_{1}=\frac{v_{i-} h_{i}\left(h^{2}\left(1-v_{i-1}\right) v_{i-1} h_{i-1}^{2} v_{i-1}^{2}\right)}{\left[-\left(1-v_{i-1}\right)^{2} h^{2}+h_{i-1}^{2} v_{i-1}^{2}\right]}
$$

$b_{2}=\frac{\left\{h_{i}\left(h^{2}\left(1-v_{i-1}\right) v_{i-1}+h_{i-1}^{2}\left(1+v_{i-1}\right) v_{i-1}+h_{i-1} 1\left\{-\left(1-v_{i}\right) \mu_{i} h^{2}+2 h_{i}^{2}\right\}\right.\right.}{\left[-\left(1-v_{i-1}\right)^{2} h^{2}+h_{i-1}^{2} v_{i-1}^{2}\right]}$

$$
b_{3}=\frac{v_{i} h_{i-1}\left\{-h^{2}\left(1-v_{i}\right)+2 h_{i}^{2}\right\}}{\left\{-\left(1-v_{i}\right)^{2} h^{2}+h_{i}^{2}\right\}} .
$$$$
\text { Clearly } k(h)=\sum a_{j}=\sum b_{j} \text {. }
$$

This theorem (4.1) is completed.

\section{CONCLUSION}

we have investigate method of Discrete Rational cubic Spline Interpolations to obtain construction, Shape, error bound and convergence.

Table 1. Given Data

$\begin{array}{llllllllllll}\boldsymbol{i} & \mathbf{1} & \mathbf{2} & \mathbf{3} & \mathbf{4} & \mathbf{5} & \mathbf{6} & \mathbf{7} & \mathbf{8} & \mathbf{9} & \mathbf{1 0} & \mathbf{1 1} \\ x_{i} & -1 & 4 & 8 & 16 & 20 & 24 & 28 & 32 & 36 & 40 & 44 \\ y_{i} & 18 & 3 & 0.05 & 1 & 4 & 8 & 15 & 24 & 34 & 40 & 43\end{array}$

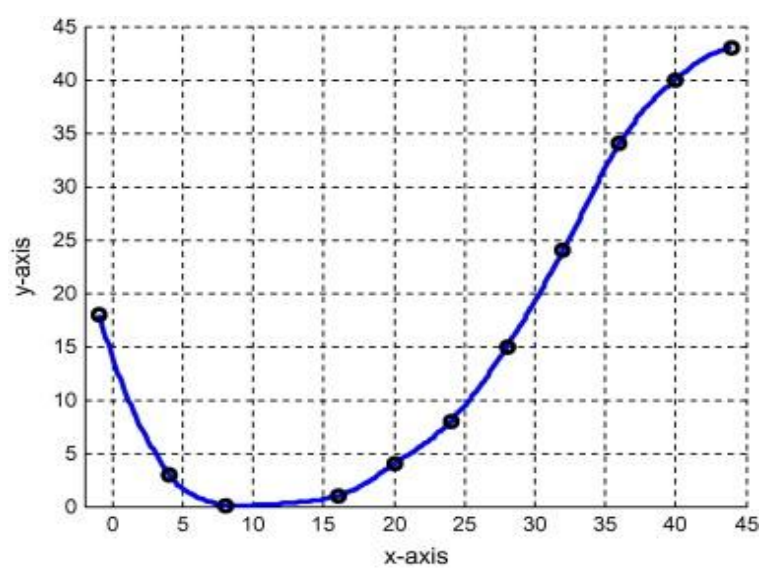

Figure 2: Rational cubic function

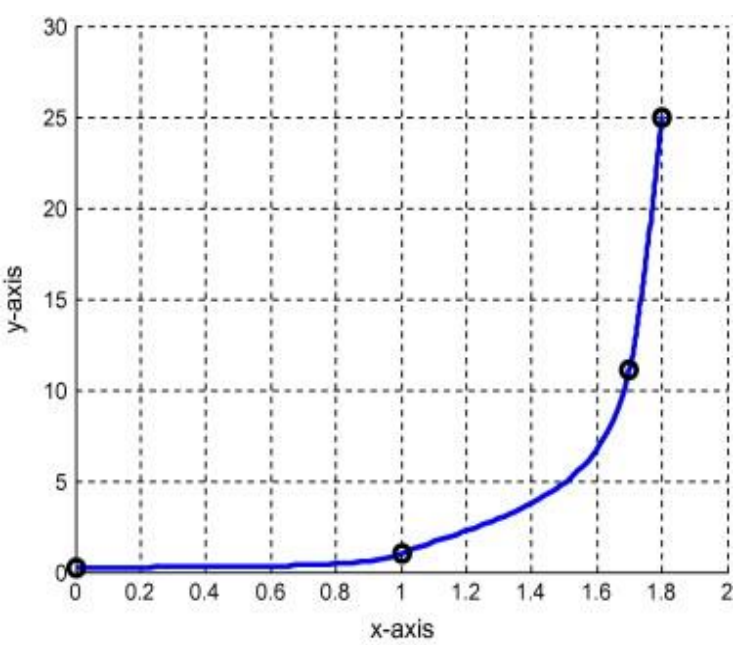

Figure 3. $C^{2}$ rational cubic curve with constant parameter

Published By:

Blue Eyes Intelligence Engineering and Sciences Publication (BEIESP) (C) Copyright: All rights reserved.

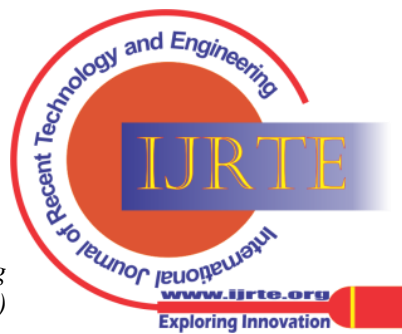




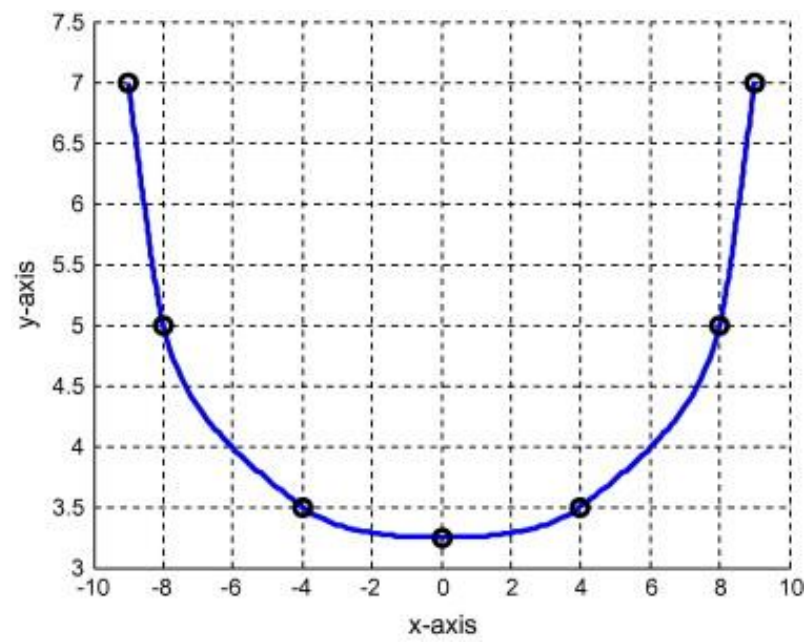

Figure 4. $C^{2}$ convex rational cubic curve

\section{ACKNOWLEDGMENT}

Thankful to Late Dr S. S. Rana Department of Mathematics and Computer Science R.D.V.V. university Jabalpur M.P. India gives suggestion to improve this paper.

\section{REFERENCES}

1. O.L. Schumaker and H.H. Schumaker - Best summation formula and discrete spline SIAM J. Anal. 10 (1973), pp, 448-459.

2. O.L. Schumaker and H.H. Schumaker, Discrete Spline Via Mathematical Programming SIAM J. Control (1971), pp, 174-183.

3. S.S. Rana and Y.P. Dubey Local Behavior of discrete cubic spline interpolation, J. Approx. Theory 86 (1996), pp. 120-127.

4. Duan Q., Wang H, Twizall E.H. A new $\mathrm{C}^{2}$ rational interpolation based on function values and constrained control of the interpolation curves. Appl.Math. Comput. 2005, 161 (2005), pp. 311-322.

5. Hussain, M.H., Sarfaraz M, Hussain M. Scientific data visualization with shape preserving $c^{1}$ rational cubic interpolation. Eur. J. Pare, Appl. Math. 2010, 3(2), pp. 194-222.

6. Sarfaraz M. Hussain M.H, Asfar N. Positive data Modeling using spline function App. Math Combutt 2010.

7. Abbas, M., A.A. Majid and J.M. Ali. Monotonicity Preserving $C^{2}$ rational cubic spline for monotone data. Applied Math. and Computation, 2019, pp- 2885-2895, (2012).

8. Karim A.A. and V.P. Kong. Local Control of the curves using rational cubic spline submitted, 2013.

9. Bao, F., Q. Sun. J. Pan and Q. Duan. Point control of the interpolating of curve with rational cubic spline. J.V.Commun Image R. 20, pp. 285-280 (2009).

10. Butt and K.W. Bodhie, Preserving positivity using piecewise cubic interpolation, Computer and Graphics 17(1) : 55-64 (1993).

11. K.W. Brodlie and S. Butt. Preserving convexity using piecewise cubic interpolation computer graphics, Vol. 15, No. 1, pp. 15-23 (1991).

12. T. Lyche. Discrete Cubic Spline Interpolation Report RRI 5, Univ. of JSIO, 1975.

\section{AUTHORS PROFILE}

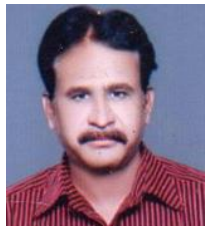

Dr. Yadvendra Prasad Dubey is Hod and Professor in Govt Kundam College Jabalpur M.P India. Fifty papers published in spline theory. Twenty-five years teaching experience in UG and PG course and four books published in Germany.



Smt Jyoti Tiwari is Assistant Professor in Mathematics Department of Shri Ram Institute of Science and Technology Jabalpur M.P. India.

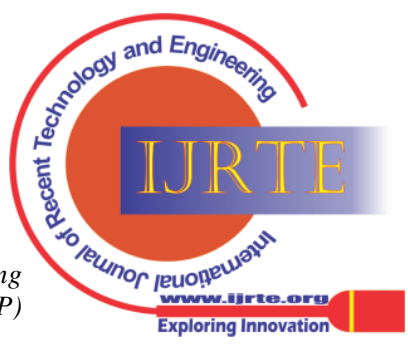

